\title{
FACIAL EXPRESSION IDENTIFICATION USING FOUR-BIT CO- OCCURRENCE MATRIXFEATURES AND K-NN CLASSIFIER
}

\author{
Bonagiri C S K Sunil Kumar ${ }^{1}$, V Bala Shankar ${ }^{2}$, Pullela S V V S R Kumar ${ }^{3}$ \\ ${ }^{1,2,3}$ Department of Computer Science \& Engineering, Aditya College of Engineering, Surampalem, East Godavari \\ District, Andhra Pradesh, India
}

\begin{abstract}
The present paper proposes a novel approach for facial expression identification based on the statistical features extracted from the 4-bit co-occurrence matrix. In the present, first color image is converted into grey level image using 7-bit quantization technique. To reduce the complexity of the Grey Level Co-occurrence matrix the present paper generates the co-occurrence matrix on 4 bit grey image which is generated by quantization technique. From The 4-bit CM extract 4 features; contrast, homogeneity, energy and correlation and generate the feature vector. By using the K-NN Classification technique classify the test image and extract the four statistical features and identify the type of expression of the test facial image. The present method got better comparative results when it is tested on Japanese Female Facial Expression (JAFFE) Database.
\end{abstract}

Key Words: Facial Expression identification, Classification, $k$-NN classifier, Quantization, and GLCM

\section{INTRODUCTION}

One of the rapidly growing fields of research is facial expression recognition due to continually growing interest in applications for automatic human behavior psychoanalysis and new technologies for human-machine announcement and multimedia retrieval. Mostly systems for Facial Expression Recognition and its Analysis proposed in the literature focused on detecting the occurrence of expressions, often using basic emotions or the Facial Action Coding System (FACS Action Units, AUs,[1]. In reality, expressions vary greatly in concentration, and concentration is often a strong cue for the explanation of the sense of expressions.

So many approaches are available in literature for the recognition of the facial expressions [2, 3]. The proposed method included the model based techniques for analyzing the face, which is considered as one global pattern without decomposing the face to different components. A feature vector, representing the expression information, is obtained by preprocessing the face image and applying different transformations for facial expression feature extraction, like Gabor wavelet [4], curvelets [5, 6], and local binary pattern $[7,8,18,19]$. Principal component analysis (PCA) is mainly used for dimensionality reduction, and multilayer neural networks or support vector machines are widely used for image classification $[9,10]$. The analytic or geometric feature based methods are used to divide the face into smaller components or subsection using which we can identify the expressions. Characteristic points are detected, and distances between Characteristic points are calculated to form the feature vectors or build a model of appearance [11, 12, 13] and support vector machines SVM [14].

The above approached methods have its own disadvantages and own demerits especially in the form of computational time and percentage of recognition. To overcome such disadvantages, the present paper derives a concept for the facial expression based on the statistical features derived from 16 gray values co-occurrence matrix. The paper is organized into 4 sections. Section 2 describe the proposed method and section 3 describes the results and discussions and conclusions are given in section 4.

\section{METHODOLOGY: FACIAL EXPRESSION RECOGNITION SYSTEM}

The proposed method mainly consists of 5 steps. In the first step, crop the given input image which covers the all facial skin of the image. In the second step, convert the cropped color image into 4-bit quantization technique. In the third step, generate the co-occurrence matrix which consists of 16 shades. Extract the 4 statistical features and generate the Feature Vector in the forth step. Based on the FV values and $\mathrm{k}-\mathrm{NN}$ classifier recognize the type of expression in the last step. The block diagram of the facial expression recognition is shown in figure 1.

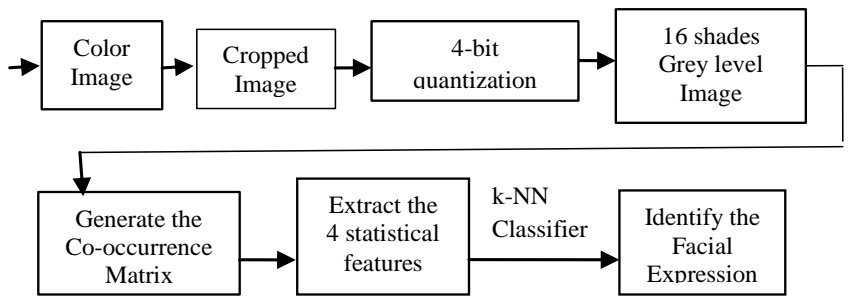

Fig -1: Block Diagram of the Facial expression Identification

Step 1 : Crop the given input image which covers the all facial skin of the image

Step 2: Crop the grey scale image: The gray facial image is cropped to cover whole skin area of the facial image. For 
efficient identification of the expression, cropping is necessary. The cropped image consists of forehead; twoeyes nose, mount, and chin area. All these appearance changed according the type of expression. Figure 2 shows an example of the original facial image and the cropped image.

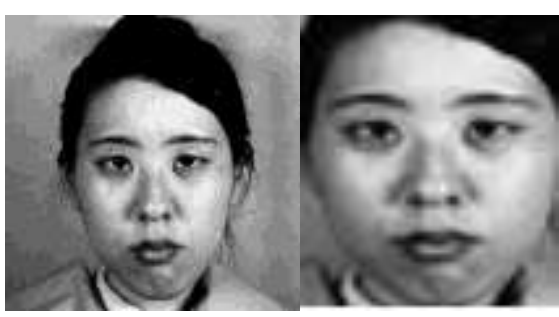

(a)

(b)

Fig -2: (a) original image (b) cropped image

Step 3: Convert the Color Image into 4-bit gray scale image using 4-bit quantization technique

The size of the Grey Level Concurrence Matrix (GLCM) depends on number of shades in the image. Generally, Gray Scale image has 256 shades and the size of the GLCM matrix is 256. So, in order to reduce the complexity of GLCM matrix, the present paper uses the 16 shades to generate the co occurrence matrix. 16 shades are represented by using 4 -bits. It can be accomplished by using quantization technique. The present paper uses 4-bit Binary Code quantization technique.

In 4-bit Binary Code quantization technique, the original images are quantized into 16shades of gray image is computed from the 256 gray shades images and then the statistical information of facial image is calculated to describe features of the image

To extract gray level features from color information, the present paper utilized the Gray shades which quantize the gray shades into 4-bins to obtain 16 gray levels. The index matrix of 16values gray image is denoted as GS(x,y). The Gray quantization process is done by using 4-bit binary code of 16 colors as follows:

$\mathrm{GS}(\mathrm{x}, \mathrm{y})=4 * \mathrm{I}($ Red $)+2 * \mathrm{I}($ Green $)+\mathrm{I}($ Blue $)$ where

$\mathrm{I}(\operatorname{Red})=0,0 \leq \operatorname{Red} \leq 16, \mathrm{I}(\operatorname{Red})=\mathrm{k}$,

$((4 * \mathrm{k})+1) \leq \operatorname{Red} \leq(4 *(\mathrm{k}+1)) \quad \mathrm{k}=[1,2,3,4]$

$\mathrm{I}($ Green $)=0,0 \leq$ Green $\leq 16, \mathrm{I}($ Green $)=\mathrm{k}$,

$((4 * \mathrm{k})+1) \leq$ Green $\leq(4 *(\mathrm{k}+1)) \quad \mathrm{k}=[1,2,3,4]$

$\mathrm{I}($ Blue $)=0,0 \leq$ Blue $\leq 16, \mathrm{I}($ Blur $)=\mathrm{k}$,

$((4 * \mathrm{k})+1) \leq$ Blue $\leq(4 *(\mathrm{k}+1)) \quad \mathrm{k}=[1,2,3,4]$
Therefore, each value of $\mathrm{GS}(\mathrm{x}, \mathrm{y})$ is a 4 bit binary code ranging from 0 to 15 . The process of 4-bit gray scale image generation is depicted in figure 3

\begin{tabular}{|l|l|l|l|l|}
\hline 139 & 128 & 131 & 134 & 132 \\
\hline 142 & 140 & 127 & 133 & 138 \\
\hline 142 & 124 & 129 & 145 & 142 \\
\hline 141 & 129 & 128 & 131 & 133 \\
\hline 137 & 134 & 134 & 137 & 137 \\
\hline
\end{tabular}

(a)

\begin{tabular}{|l|l|l|l|l|}
\hline 9 & 8 & 8 & 8 & 8 \\
\hline 9 & 9 & 8 & 8 & 9 \\
\hline 9 & 8 & 8 & 9 & 9 \\
\hline 9 & 8 & 8 & 8 & 8 \\
\hline 9 & 8 & 8 & 9 & 9 \\
\hline
\end{tabular}

(b)

Fig -3: 4-bit quantization procedure a) Sample Gray level Image b) Generated 4-bit 16 shade gray image

Step 4: generate the 4 bit grey scale concurrence matrix: Grey level co-occurrence matrix (GLCM) is the one of the simplest technique for describing texture image[15]. The GLCM is gives complete and detailed information about the image. Generally the size of co-occurrence matrix of image number of gray level on the image. For Generating GLCM of the Gray level image, the size of the GLCM is $256 \times 256$. This I is the one of the drawback of GLCM. It takes more computational time for generating the co-occurrence matrix. To avoid such problem, the present paper generates the cooccurrence matrix on 16 shades image so that the size of the GLCM is reduced to $16 \times 16$ and its causes reduces the computational cost drastically. The generated matrix called Four-bit Co-occurrence Matrix (FCoM).

A set of statistical features defined by Haralick [15] are extracted on FCoM of the facial image. The features used in this method are energy, contrast, homogeneity, and correlation. The equations of the considered feature are shown in equations 1 to 4 . The proposed FCoM combines the merits of both statistical computation cost for processing of the facial images. The GLCM gives the whole information of the facial expression image

$$
\begin{aligned}
& \text { contrast }=\sum_{\mathrm{i} j=0}^{\mathrm{N}-1}-\ln \left(\mathrm{P}_{\mathrm{ij}}\right)^{2} \\
& \text { Homogenity }=\sum_{\mathrm{i}, \mathrm{j}=0}^{\mathrm{N}-1} \frac{\mathrm{P}_{\mathrm{ij}}}{1+(\mathrm{i}-\mathrm{j})^{2}} \\
& \text { Correlation }=\sum_{\mathrm{i}, \mathrm{j}=0}^{\mathrm{N}-1} \mathrm{P}_{\mathrm{ij}} \frac{(\mathrm{i}-\mu)(\mathrm{j}-\mu)}{\sigma^{2}} \\
& \text { Energy }=\sum_{\mathrm{i}, \mathrm{j}=0}^{\mathbb{N}-1}-\ln \left(\mathrm{P}_{\mathrm{ij}}\right)^{2}
\end{aligned}
$$

\section{RESULTS AND DISCUSSIONS}

To prove the functionality of the proposed scheme, it has established a database contains 213 facial expression images of females from Japanese Female Facial Expression (JAFFE) Database. The Database was collected by Kamachi and Gyoba at Kyushu University[16], Japan. Original 
images have been rescaled and cropped such that the eyes are roughly at the same position within a distance of 60 pixels in the final images (resolution: $256 \times 256$ pixels). The images include the seven categories of expressions (neutral, sadness, surprise, happiness, anger, disgust and fear) is roughly the same. A few of them are shown in Figure. 4.
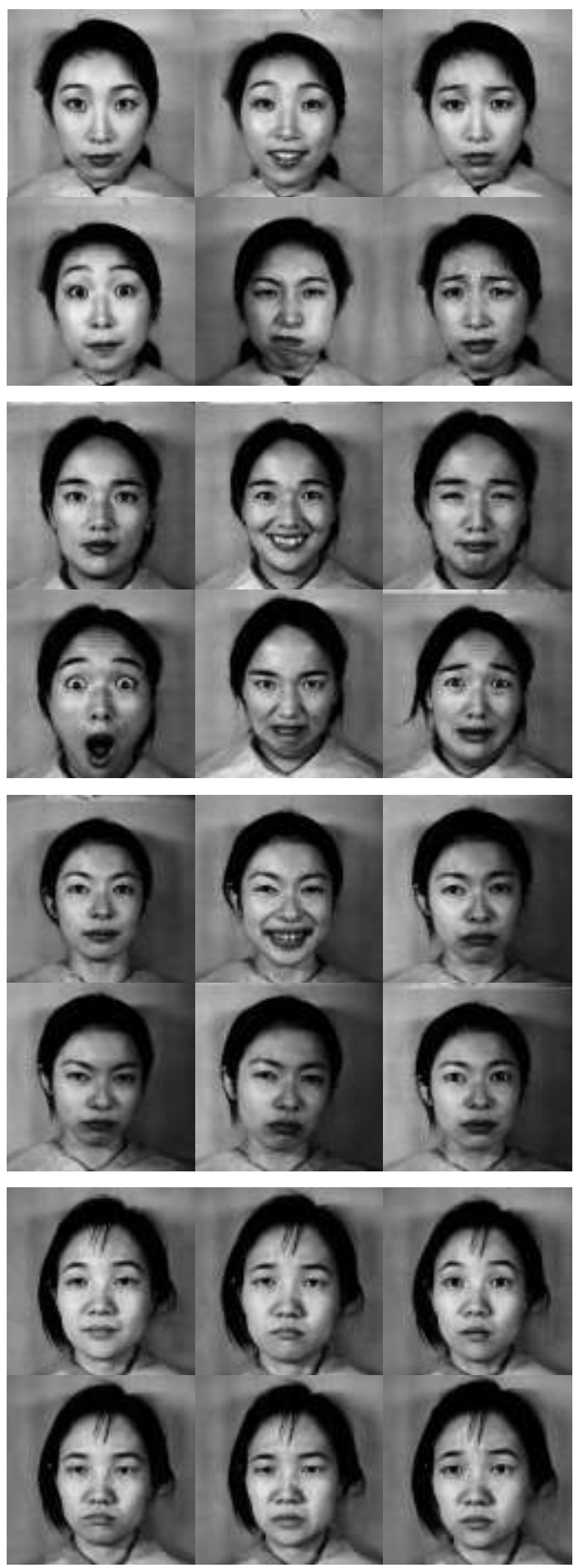

Fig -4: Facial expression database: Examples

In the proposed method the test images are grouped into seven categories based on expression (neutral, sadness, surprise, happiness, disgust, anger, and fear). The four statistical features are extracted from JAFFE database and generate the feature vector. FCoM of seven category results are stored in the feature vector. Feature vector directs to demonstration of features of the FAFFE images. The statistical features of seven groups of facial expressions are shown in tables $1,2,3,4,5,6$, and 7 respectively. To prove the efficiency of the proposed method, took100 different facial expressions from JAFFE data base, Google database and scanned images and extracted the statistical features from those images and the results are stored in the test vector. Estimate the minimum distance between feature vector and test vector and stored the result in the library. Classify the test images into appropriate expression category using minimum distance K-nearest neighbor classifier. Some of the successful classification results of test data based on the proposed method are shown in table 8 . The classification results of the proposed method are shown in table 9 and corresponding classification graph is shown in figure 5 .

Table 1: Feature set values of Anger expression images

\begin{tabular}{|c|c|c|c|c|c|}
\hline $\begin{array}{l}\text { S. } \\
\text { No }\end{array}$ & $\begin{array}{l}\text { Image } \\
\text { Name }\end{array}$ & $\begin{array}{c}\text { Correla- } \\
\text { tion }\end{array}$ & Contrast & Energy & Homogeneity \\
\hline 1 & Img.104 & -0.0358 & 32.33 & 0.028 & 0.2128 \\
\hline 2 & Img.105 & -0.0317 & 31.39 & 0.028 & 0.2117 \\
\hline 3 & Img.106 & -0.0683 & 31.67 & 0.033 & 0.2097 \\
\hline 4 & Img. 125 & 0.0313 & 30.27 & 0.035 & 0.2404 \\
\hline 5 & Img.127 & 0.0499 & 30.75 & 0.032 & 0.2439 \\
\hline 6 & Img.146 & 0.0306 & 30.14 & 0.032 & 0.2407 \\
\hline 7 & Img.147 & 0.0387 & 29.93 & 0.027 & 0.2315 \\
\hline 8 & Img.148 & 0.0496 & 29.47 & 0.027 & 0.2280 \\
\hline 9 & Img.167 & 0.0013 & 33.94 & 0.037 & 0.2421 \\
\hline 10 & Img. 168 & 0.0120 & 32.72 & 0.035 & 0.2350 \\
\hline 11 & Img.169 & 0.0117 & 32.61 & 0.032 & 0.2375 \\
\hline 12 & Img.17 & 0.0518 & 29.65 & 0.038 & 0.2616 \\
\hline 13 & Img.18 & 0.0568 & 30.45 & 0.036 & 0.2459 \\
\hline 14 & Img.19 & 0.0621 & 30.19 & 0.043 & 0.2627 \\
\hline 15 & Img.190 & 0.0595 & 29.75 & 0.033 & 0.2477 \\
\hline 16 & Img.191 & 0.0577 & 30.92 & 0.035 & 0.2479 \\
\hline 17 & Img.192 & 0.0484 & 30.58 & 0.034 & 0.2439 \\
\hline 18 & Img. 211 & 0.0175 & 32.86 & 0.037 & 0.2344 \\
\hline 19 & Img. 212 & 0.0560 & 31.45 & 0.037 & 0.2503 \\
\hline 20 & Img.213 & 0.0582 & 30.83 & 0.032 & 0.2492 \\
\hline
\end{tabular}


Table 2: Feature set values of Disgust expression images

\begin{tabular}{|c|c|c|c|c|c|}
\hline $\begin{array}{c}\mathrm{S} \\
\text { No }\end{array}$ & $\begin{array}{l}\text { Image } \\
\text { Name }\end{array}$ & Correlation & Contrast & Homogeneity & Energy \\
\hline 1 & Img 1.193 & 0.086 & 28.150 & 0.273 & 0.033 \\
\hline 2 & Img 86 & -0.183 & 34.870 & 0.179 & 0.039 \\
\hline 3 & Img.107 & -0.057 & 30.060 & 0.198 & 0.036 \\
\hline 4 & Img. 128 & 0.032 & 29.390 & 0.235 & 0.037 \\
\hline 5 & Img. 129 & 0.031 & 29.560 & 0.240 & 0.037 \\
\hline 6 & Img.130 & 0.047 & 30.800 & 0.244 & 0.038 \\
\hline 7 & Img. 149 & 0.015 & 28.173 & 0.225 & 0.036 \\
\hline 8 & Img. 150 & 0.096 & 26.264 & 0.242 & 0.041 \\
\hline 9 & Img. 151 & 0.078 & 27.842 & 0.249 & 0.039 \\
\hline 10 & Img. 170 & 0.015 & 31.270 & 0.245 & 0.038 \\
\hline 11 & Img. 171 & 0.002 & 32.820 & 0.231 & 0.043 \\
\hline 12 & Img.172 & -0.037 & 32.520 & 0.221 & 0.043 \\
\hline 13 & Img. 173 & 0.032 & 32.030 & 0.244 & 0.040 \\
\hline 14 & Img.194 & 0.063 & 27.168 & 0.257 & 0.036 \\
\hline 15 & Img.195 & 0.018 & 29.784 & 0.238 & 0.040 \\
\hline 16 & Img.20 & 0.018 & 30.520 & 0.245 & 0.044 \\
\hline 17 & Img. 215 & 0.067 & 30.150 & 0.240 & 0.040 \\
\hline 18 & Img. 216 & 0.045 & 30.400 & 0.244 & 0.039 \\
\hline 19 & Img.22 & 0.082 & 27.590 & 0.255 & 0.044 \\
\hline 20 & Img.42 & 0.038 & 29.449 & 0.245 & 0.036 \\
\hline
\end{tabular}

Table 3: Feature set values of Disgust expression images

\begin{tabular}{|c|c|c|c|c|c|}
\hline Sno & $\begin{array}{c}\text { Image } \\
\text { Name }\end{array}$ & Correlation & Contrast & Homogeneity & Energy \\
\hline 1 & img 117 & -0.219 & 29.82 & 0.216 & 0.033 \\
\hline 2 & img 118 & -0.247 & 30.83 & 0.216 & 0.034 \\
\hline 3 & img 137 & -0.154 & 28.36 & 0.242 & 0.034 \\
\hline 4 & img 138 & -0.124 & 28.09 & 0.247 & 0.036 \\
\hline 5 & img 139 & -0.192 & 29.73 & 0.236 & 0.032 \\
\hline 6 & img 202 & -0.202 & 31.43 & 0.236 & 0.035 \\
\hline 7 & img 203 & -0.23 & 30.89 & 0.23 & 0.034 \\
\hline 8 & img 204 & -0.28 & 29.6 & 0.201 & 0.031 \\
\hline 9 & img132 & -0.167 & 31.17 & 0.245 & 0.038 \\
\hline 10 & img152 & -0.089 & 27.78 & 0.277 & 0.042 \\
\hline 11 & img153 & -0.138 & 29.84 & 0.251 & 0.041 \\
\hline 12 & img159 & -0.21 & 31.87 & 0.234 & 0.038 \\
\hline 13 & img160 & -0.21 & 31.79 & 0.23 & 0.035 \\
\hline 14 & img180 & -0.197 & 30.51 & 0.24 & 0.035 \\
\hline 15 & img181 & -0.211 & 30.67 & 0.226 & 0.031 \\
\hline 16 & img182 & -0.149 & 29.69 & 0.259 & 0.036 \\
\hline 17 & img21 & -0.219 & 31.14 & 0.211 & 0.031 \\
\hline 18 & img30 & -0.203 & 30.71 & 0.218 & 0.034 \\
\hline 19 & img31 & -0.222 & 31.19 & 0.217 & 0.031 \\
\hline & & & & & \\
\hline
\end{tabular}

Table 4: Feature set values of Netural expression images

\begin{tabular}{|c|c|c|c|c|c|}
\hline Sno & $\begin{array}{l}\text { Image } \\
\text { Name }\end{array}$ & Correlation & Contrast & Homogeneity & Energy \\
\hline 1 & img 1 & 0.0514 & 30.76 & 0.254 & 0.044 \\
\hline 2 & img 113 & -0.0336 & 30.78 & 0.219 & 0.039 \\
\hline 3 & img 114 & 0.0034 & 30.24 & 0.222 & 0.037 \\
\hline 4 & img 115 & -0.0436 & 30.68 & 0.212 & 0.035 \\
\hline 5 & img 134 & 0.0884 & 27.55 & 0.264 & 0.045 \\
\hline 6 & img 135 & 0.1134 & 27.34 & 0.272 & 0.046 \\
\hline 7 & img 136 & 0.1104 & 26.24 & 0.266 & 0.047 \\
\hline 8 & img 155 & 0.0114 & 30.7 & 0.248 & 0.045 \\
\hline 9 & img 156 & 0.0344 & 31.71 & 0.24 & 0.042 \\
\hline 10 & $\operatorname{img} 157$ & -0.0036 & 30.4 & 0.224 & 0.033 \\
\hline 11 & img 199 & -0.0636 & 29.86 & 0.202 & 0.033 \\
\hline 12 & $\operatorname{img} 2$ & 0.0784 & 29.39 & 0.25 & 0.04 \\
\hline 13 & img 200 & -0.0216 & 31.22 & 0.222 & 0.035 \\
\hline 14 & img 201 & -0.0126 & 28.78 & 0.212 & 0.032 \\
\hline 15 & img 26 & 0.0464 & 30.56 & 0.236 & 0.037 \\
\hline 16 & img 27 & -0.0146 & 31.78 & 0.222 & 0.037 \\
\hline 17 & $\operatorname{img} 28$ & -0.0366 & 31.83 & 0.214 & 0.038 \\
\hline 18 & $\operatorname{img} 3$ & 0.1184 & 28.13 & 0.268 & 0.047 \\
\hline 19 & img 49 & 0.1374 & 28.26 & 0.288 & 0.054 \\
\hline 20 & img 50 & 0.1354 & 27.34 & 0.295 & 0.057 \\
\hline
\end{tabular}

Table 5: feature set values of Sadness expression images

\begin{tabular}{|l|l|l|l|l|l|}
\hline Sno & $\begin{array}{c}\text { Image } \\
\text { Name }\end{array}$ & Contrast & Correlation & Energy & Homogeneity \\
\hline 1 & img 10 & 29.14 & 0.0585 & 0.046 & 0.259 \\
\hline 2 & img 100 & 30.6 & -0.0445 & 0.033 & 0.203 \\
\hline 3 & img 11 & 27.9 & 0.0075 & 0.035 & 0.224 \\
\hline 4 & img 119 & 30.38 & 0.0155 & 0.041 & 0.228 \\
\hline 5 & img 12 & 28.76 & 0.0035 & 0.039 & 0.236 \\
\hline 6 & img 120 & 29.8 & -0.0605 & 0.036 & 0.212 \\
\hline 7 & img 121 & 30.54 & -0.0005 & 0.039 & 0.224 \\
\hline 8 & img 140 & 27.19 & 0.0975 & 0.036 & 0.248 \\
\hline 9 & img 141 & 28.01 & 0.0585 & 0.033 & 0.232 \\
\hline 10 & img 142 & 28.67 & 0.0395 & 0.039 & 0.237 \\
\hline 11 & img 161 & 31.21 & 0.0295 & 0.043 & 0.248 \\
\hline 12 & img 162 & 31.34 & 0.0335 & 0.043 & 0.245 \\
\hline 13 & img 163 & 29.55 & 0.0615 & 0.041 & 0.251 \\
\hline 14 & img 184 & 28.99 & -0.0195 & 0.036 & 0.232 \\
\hline 15 & img 185 & 29.86 & 0.0505 & 0.04 & 0.248 \\
\hline 16 & img 186 & 29.74 & 0.0605 & 0.041 & 0.255 \\
\hline 17 & img 205 & 30.02 & 0.0365 & 0.038 & 0.236 \\
\hline 18 & img 206 & 29.53 & 0.0705 & 0.037 & 0.251 \\
\hline 19 & img 207 & 30.82 & -0.0185 & 0.034 & 0.218 \\
\hline 20 & img 33 & 31.12 & -0.0195 & 0.037 & 0.23 \\
\hline
\end{tabular}


Table 6: Feature set values of fear expression images

\begin{tabular}{|l|l|l|l|l|l|}
\hline Sno & \multicolumn{1}{|l|}{$\begin{array}{l}\text { Name } \\
\text { Name }\end{array}$} & Correlation & Contrast & Homogeneity & Energy \\
\hline 1 & img 174 & -0.003 & 31.78 & 0.242 & 0.042 \\
\hline 2 & img 175 & 0.056 & 30.38 & 0.247 & 0.044 \\
\hline 3 & img 176 & -0.023 & 32.2 & 0.242 & 0.044 \\
\hline 4 & img110 & -0.057 & 30.91 & 0.213 & 0.036 \\
\hline 5 & img111 & -0.07 & 30.81 & 0.205 & 0.033 \\
\hline 6 & img112 & -0.079 & 31.92 & 0.207 & 0.038 \\
\hline 7 & img131 & 0.067 & 29.54 & 0.243 & 0.041 \\
\hline 8 & img132 & 0.02 & 30.49 & 0.231 & 0.038 \\
\hline 9 & img133 & 0.022 & 31.52 & 0.244 & 0.044 \\
\hline 10 & img152 & 0.044 & 28.75 & 0.253 & 0.041 \\
\hline 11 & img153 & 0.088 & 28.32 & 0.253 & 0.039 \\
\hline 12 & img154 & 0.089 & 27.65 & 0.255 & 0.041 \\
\hline 13 & img196 & 0.1 & 29.03 & 0.262 & 0.041 \\
\hline 14 & img197 & 0.121 & 28.37 & 0.272 & 0.045 \\
\hline 15 & img198 & 0.058 & 30.41 & 0.263 & 0.047 \\
\hline 16 & img217 & -0.025 & 32.01 & 0.223 & 0.038 \\
\hline 17 & img218 & 0.006 & 31.04 & 0.23 & 0.037 \\
\hline 18 & img219 & 0.03 & 29.77 & 0.244 & 0.036 \\
\hline 19 & img23 & 0.064 & 30.6 & 0.262 & 0.046 \\
\hline 20 & img24 & 0.013 & 28.32 & 0.227 & 0.038 \\
\hline
\end{tabular}

Table 7: Feature set values of Surprise expression images

\begin{tabular}{|c|c|c|c|c|c|}
\hline Sno & $\begin{array}{c}\text { Image } \\
\text { Name }\end{array}$ & Correlation & Contrast & Homogeneity & Energy \\
\hline 1 & img101 & -0.074 & 31.7 & 0.2 & 0.034 \\
\hline 2 & img102 & -0.083 & 31.63 & 0.194 & 0.032 \\
\hline 3 & img103 & -0.044 & 30.65 & 0.206 & 0.034 \\
\hline 4 & img122 & -0.049 & 30.96 & 0.21 & 0.036 \\
\hline 5 & img123 & 0.01 & 30.69 & 0.229 & 0.038 \\
\hline 6 & img124 & -0.031 & 30.49 & 0.222 & 0.036 \\
\hline 7 & img14 & 0.048 & 30.51 & 0.246 & 0.043 \\
\hline 8 & img143 & 0.055 & 29.27 & 0.243 & 0.038 \\
\hline 9 & img144 & 0.058 & 28.02 & 0.246 & 0.04 \\
\hline 10 & img145 & 0.039 & 29.07 & 0.234 & 0.035 \\
\hline 11 & img15 & 0.037 & 30.39 & 0.253 & 0.045 \\
\hline 12 & img16 & 0.063 & 29.42 & 0.253 & 0.042 \\
\hline 13 & img164 & 0.037 & 30.03 & 0.239 & 0.038 \\
\hline 14 & img165 & 0.019 & 31.08 & 0.232 & 0.04 \\
\hline 15 & img166 & 0.047 & 31.01 & 0.236 & 0.041 \\
\hline 16 & img187 & 0.032 & 29.05 & 0.244 & 0.039 \\
\hline 17 & img188 & 0.046 & 28.84 & 0.249 & 0.04 \\
\hline 18 & img189 & 0.041 & 29.58 & 0.239 & 0.039 \\
\hline 19 & img208 & -0.03 & 32.08 & 0.22 & 0.038 \\
\hline 20 & img209 & 0.045 & 30.14 & 0.236 & 0.037 \\
\hline
\end{tabular}

Table 8: Feature values of Test database images

\begin{tabular}{|c|l|c|c|c|c|c|c|}
\hline Sno & Image Name & Correlation & Contrast & Homogeneity & Energy & Group & Result \\
\hline 1 & ing190 & -0.11366 & 34.4 & 0.198 & 0.033 & Anger & correct \\
\hline 2 & img144 & -0.03 & 32.08 & 0.22 & 0.038 & Surprise & correct \\
\hline 3 & img156 & -0.0126 & 28.78 & 0.212 & 0.032 & Neutral & correct \\
\hline 4 & gog_im1 & -0.074 & 31.7 & 0.2 & 0.034 & Surprise & correct \\
\hline 5 & gog_im2 & 0.0395 & 28.67 & 0.237 & 0.039 & Neutral & correct \\
\hline 6 & gog_im3 & -0.0195 & 28.99 & 0.232 & 0.036 & Sadness & correct \\
\hline 7 & gog_im4 & 0.063164 & 29.32 & 0.248 & 0.036 & Disgust & correct \\
\hline 8 & gog_im5 & -0.179 & 28.82 & 0.221 & 0.028 & Happiness & correct \\
\hline 9 & si-08 & -0.003 & 31.78 & 0.242 & 0.042 & Fear & correct \\
\hline 10 & si-09 & 0.066517 & 29.99 & 0.24 & 0.04 & Disgust & correct \\
\hline 11 & si-10 & -0.21 & 31.79 & 0.23 & 0.035 & Happiness & correct \\
\hline 12 & si-11 & -0.023 & 32.2 & 0.242 & 0.044 & Fear & correct \\
\hline 13 & img140 & 0.0035 & 28.76 & 0.236 & 0.039 & Sadness & correct \\
\hline 14 & img65 & 0.001981 & 32.28 & 0.23 & 0.043 & Anger & correct \\
\hline 15 & img204 & -0.247 & 30.83 & 0.216 & 0.034 & Happiness & correct \\
\hline 16 & img131 & 0.06 & 28.65 & 0.261 & 0.045 & Fear & correct \\
\hline 17 & gog_im11 & 0.045202 & 30.042 & 0.244 & 0.039 & Disgust & correct \\
\hline 18 & gog_im12 & -0.209 & 30.19 & 0.215 & 0.032 & Happiness & correct \\
\hline 19 & si-01 & 0.0784 & 29.39 & 0.25 & 0.04 & Neutral & correct \\
\hline 20 & gog_im6 & -0.003 & 31.78 & 0.242 & 0.042 & Fear & correct \\
\hline 21 & gog_im7 & -0.0486 & 30.59 & 0.209 & 0.035 & Neutral & correct \\
\hline 22 & gog_im8 & -0.03165 & 31.93 & 0.211 & 0.028 & Anger & correct \\
\hline 23 & gog_im9 & 0.037 & 30.03 & 0.239 & 0.038 & Surprise & correct \\
\hline 24 & gog_im10 & 0.0344 & 31.71 & 0.24 & 0.042 & Neutral & correct \\
\hline 25 & si-02 & 0.049949 & 30.57 & 0.243 & 0.032 & Anger & correct \\
\hline
\end{tabular}


Table 9: Classification Results of the test database

\begin{tabular}{|c|c|c|c|c|}
\hline $\begin{array}{c}\text { Data } \\
\text { Base } \\
\text { Name }\end{array}$ & $\begin{array}{c}\text { No of } \\
\text { Images }\end{array}$ & $\begin{array}{c}\text { Correctly } \\
\text { Classified }\end{array}$ & $\begin{array}{c}\text { Not } \\
\text { correctly } \\
\text { classified }\end{array}$ & $\begin{array}{c}\% \\
\text { recognition }\end{array}$ \\
\hline JAFFE & 60 & 59 & 1 & 98.33 \\
\hline Google & 20 & 19 & 1 & 95.00 \\
\hline Scanned & 20 & 19 & 1 & 95.00 \\
\hline
\end{tabular}

\section{CONCLUSIONS}

The present paper proposed a new scheme for generating the gray scale image with 16 shades. The novelty of the present approach is that its take less time to generate the cooccurrence matrix because of the image has only 16 gray levels. The proposed method has got the \%recognition is about 96.11 when applied on 3 different databases.

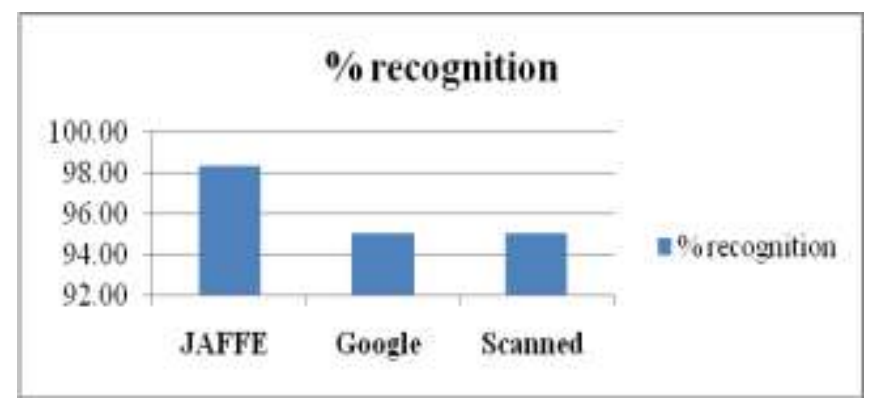

Fig -5: Classification graph of the proposed method

\section{REFERENCES}

[1] M. Bartlett, G. Littlewort, M. Frank, C. Lainscsek, I. Fasel, and J. Movellan. Automatic recognition of facial actions in spontaneous expressions. Journal of Multimedia, 1(6):22-35, 2006.

[2] B. Fasel and J. Luettin. Automatic facial expression analysis: a survey.Pattern Recognition, 36(1):259-275, 2003.

[3] Z. Zeng, M. Pantic, G. I. Roisman, and T. S. Huang. A survey of affectrecognition methods: audio, visual, and spontaneous expressions.IEEE Trans. Pattern Analysis nd Machine Intelligence, 31(1):39-58,2009

[4] Juliano J. Bazzo Marcus V. Lamar, "Recognizing Facial Actions Using Gabor Wavelets with Neutral Face Average Difference," Sixth IEEE International Conference on Automatic Face and Gesture Recognition (FGR'04), 2004.

[5] Xianxing $\mathrm{Wu}$ and Jieyu Zhao, "Curvelet Feature Extraction for Face Recognition and Facial Expression Recognition", Sixth International

[6] Conference on Natural Computation, pp.1212-1216, 2010

[7] E. Candés, L. Demanet, D. Donoho and L. Ying, "Fast Discrete Curvelet Transforms,"Applied and Computational Mathematics, Caltech, Pasadena, 2006.

[8] S. Singh and R. Maurya, A. Mittal,"Application of Complete Local Binary Pattern Method for Facial Expression Recognition", IEEE Proceedings of 4th International Conference on Intelligent Human Computer Interaction, Kharagpur, India, December 2729, 2012.
[9] R.Hablani,N. Chaudhari andS.Tanwani "Recognition of Facial Expressions using Local Binary Patternsof Important Facial Parts," International Journal of Image Processing (IJIP), vol. 7 (2), 2013.

[10] M.Pantic and L.J.M. Rothkrantz, "Expert System For Automatic Analysis Of Facial Expressions," Image And Vision Computing,vol.18, pp. 881-905,2000.

[11]D. Arumugan, S. Purushothaman."Emotion Classification Using Facial Expression," International Journal of advanced Computer Science and Application, vol. 2, no 7,2011.

[12] T. F. Cootes, G. J. Edwards and C. J. Taylor. "Active Appearance Models," IEEE Transaction on Pattern Analysis and Machine Intelligence, vol. 23, n 6, 2001.

[13] M. S. Bartlett, J. C. Hager, P. Ekman, and T. J. Sejnowski."Measuring facial expressions by computer image analysis," Psychophysiology, Cambridge University, USA, vol. 36, pp. 253-263, 1999.

[14]Zheng You. "Feature-Based Facial Expression Recognition: Sensitivity Analysis and Experiments with a Multi-Layer Perceptron," International Journal of pattern Recognition and artificial Intelligence vol. 13(6), pp. 893-911, 1999.

[15]M. S. Bartlett, J. C. Hager, P. Ekman, and T. J. Sejnowski."Measuring facial expressions by computer image analysis, "Psychophysiology, Cambridge University, USA, vol. 36, pp. 253-263, 1999.

[16] Robert M. Haralick, K Shanmugam and Its'Hak Dinstein(1979). "Textural Features for Image Classification." IEEE Transactions on Systems, Man, and Cybernetics pp. 610-621.

[17] M. Lyons, S. Akamatsu, M. Kamachi, and J. Gyoba. Coding facial expressions with gabor wavelets. In Proceedings of the Third IEEE International Conference on Automatic Face and Gesture Recognition, Nara, Japan, Apr. 1998

[18]SVVSR Kumar, Pullela., V.Vijaya Kumar, and Rampay. Venkatarao. "Age Classification Based On Integrated Approach", International Journal of Image Graphics and Signal Processing, 2014

[19] Satyanarayana Murty, Gorti, J Sasi Kiran, and V.Vijaya Kumar. "Facial Expression Recognition Based on Features Derived From the Distinct LBP and GLCM", International Journal of Image Graphics and Signal Processing, 2014.

[20] Pullela S V V S R Kumar et.al., Classification of Facial Expressions based on Transitions Derived from Third Order Neighborhood LBP, Global Journal of Computer Science and Technology, Graphics \& Vision, Vol. 14 no. 1, pp. 1-12, 2014.

\section{BIOGRAPHIES}

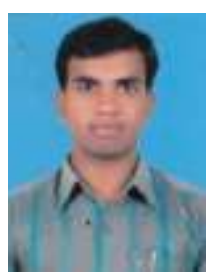

Bonagiri C S K Sunil Kumar is pursuing his masters degree from Aditya College of Engineering, Surampalem. His research interests include Data Mining and Image processing. 


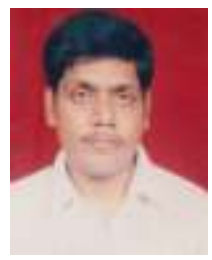

V Bala Shankar is working as Senior Assistant Professor in the Department of Computer Science \& Engineering, Aditya College of Engineering, Surampalem. His research interests include Image Processing, Cloud Computing.

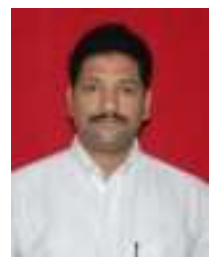

Dr. Pullela S V V S R Kumar is working as Professor in CSE at Aditya College of Engineering, Surampalem. He received his Ph.D degree from Acharya Nagarjuna University. He is having more than 15 years of experience and published 13 research papers in various International Journals and Conferences. He acted as a reviewer and PC Member for various international conferences. His research interests include Data Mining, Pattern Recognition and Image Processing. 